\title{
Immunohistochemical Expression and Clinical Significance of Suggested Stem Cell Markers in Hepatocellular Carcinoma
}

Jong Jin Sung · Sang Jae Noh Jun Sang Bae $\cdot$ Ho Sung Park Kyu Yun Jang · Myoung Ja Chung Woo Sung Moon

Department of Pathology, Research Institute of Clinical Medicine of Chonbuk National University, Biomedical Research Institute of Chonbuk National University Hospital and Research Institute for Endocrine Sciences, Chonbuk National University Medical School, Jeonju, Korea

Received: August 28, 2015

Accepted: October 9, 2015

\section{Corresponding Author}

Woo Sung Moon, MD, PhD

Department of Pathology, Chonbuk National

University Medical School, 567 Baekje-daero,

Deokjin-gu, Jeonju 54896, Korea

Tel: +82-63-270-3086

Fax: +82-63-270-3135

E-mail: mws@chonbuk.ac.kr

\begin{abstract}
Background: Increasing evidence has shown that tumor initiation and growth are nourished by a small subpopulation of cancer stem cells (CSCs) within the tumor mass. CSCs are posited to be responsible for tumor maintenance, growth, distant metastasis, and relapse after curative operation. We examined the expression of CSC markers in paraffin-embedded tissue sections of hepatocellular carcinoma (HCC) and correlated the results with clinicopathologic characteristics. Methods: Immunohistochemical staining for the markers believed to be expressed in the CSCs, including epithelial cell adhesion molecule (EpCAM), keratin 19 (K19), CD133, and CD56, was performed in $82 \mathrm{HCC}$ specimens. Results: EpCAM expression was observed in $56 \%$ of the HCCs (46/82) and K19 in 6\% (5/82). EpCAM expression in HCC significantly correlated with elevated $\alpha$-fetoprotein level, microvessel invasion of tumor cells, and high histologic grade. In addition, EpCAM expression significantly correlated with K19 expression. The overall survival and relapsefree survival rates in patients with EpCAM-expressing $\mathrm{HCC}$ were relatively lower than those in patients with EpCAM-negative HCC. All but two of the 82 HCCs were negative for CD133 and CD56, respectively. Conclusions: Our results suggest that HCCs expressing EpCAM are associated with unfavorable prognostic factors and have a more aggressive clinical course than those not expressing EpCAM. Further, the expression of either CD133 or CD56 in paraffin-embedded HCC tissues appears to be rare.
\end{abstract}

Key Words: Carcinoma, hepatocellular; EpCAM protein; Cancer stem cells
Hepatocellular carcinoma (HCC) is recognized worldwide as the fifth most common solid cancer and the third leading cause of cancer-associated mortality. ${ }^{1}$ Although remarkable advancement in the treatment of HCC has been made over the past few decades, HCC is still related to a high rate of mortality because of its recurrence and metastasis. ${ }^{2}$ Increasing evidence has demonstrated that tumor maintenance and growth are nourished by a small subpopulation of cancer stem cells (CSCs) within the tumor mass. CSCs are hypothesized to be responsible for tumor initiation, recurrence, generation of distant metastases, and resistance to radiation and chemotherapy. ${ }^{3-7}$ Recent research on HCC has centered on the issues of CSCs, which include identification of CSCs, expansion of CSC markers, and therapeutic targeting of $\mathrm{CSCs}^{7-11}$ since CSC markers might be useful for estimating the prognosis of HCC patients. Several markers including epithelial cell adhesion molecule (EpCAM) ${ }^{8}$ keratin 19 (K19), ${ }^{9} \mathrm{CD} 133,{ }^{10}$ and $\mathrm{CD} 56^{11}$ have been proposed as CSC markers in HCC, and they are termed stemness markers.
In the present study, the location and expression of four suggested CSC markers, namely EpCAM, K19, CD133, and CD56, were examined in paraffin-embedded tissue sections of 82 HCCs, and the relationships between expression of these markers and clinicopathologic characteristics of HCC were investigated.

\section{MATERIALS AND METHODS}

\section{Patients and specimens}

This study was approved by the Institutional Review Board (IRB) of Chonbuk National University Hospital. Informed consent was obtained from all patients who underwent surgery according to the Helsinki Declaration. To examine the location and expression of four suggested CSC markers, we collected 91 surgical specimens of formalin-fixed, paraffin-embedded HCCs resected in the Department of Pathology, Chonbuk National University Hospital, between January 2011 and December 2013. Among these patients, nine underwent transarterial chemoem- 
bolization, which resulted in near total necrosis of the tissue. The remaining 82 patients were analyzed in our study. In each case, clinicopathologic findings, including age, gender, etiology, background liver disease, ascites, serological data including serum albumin level and $\alpha$-fetoprotein (AFP) level, microvessel invasion, intrahepatic metastasis, histologic grade, and follow-up data, were obtained from hospital records. Tumors were staged according to the criteria of the 2010 American Joint Committee on Cancer (AJCC) TNM classification. ${ }^{12}$ Follow-up period was defined as the period from the date of initial surgery to the date of either last follow-up or death.

\section{Immunohistochemistry}

For immunohistochemical (IHC) staining, 10\% formalinfixed, paraffin-embedded tissue sections of representative areas of tumor were prepared into 4 - $\mu$ m-thick tissue samples. IHC staining was performed using a fully automated IHC system with the Bond Polymer Refine Detection Kit (Leica Bond, Newcastle upon Tyne, UK) according to the manufacturer's instructions. The sources of antibodies used in this study and conditions of the procedure are listed in Table 1. Peroxidase activity was detected with the enzyme substrate 3-amino-9-ethyl carbazole. The immunoreactivity of the specimens was interpreted according to the intensity of staining and proportion of positive cells. The intensity of cytoplasmic and membranous staining was graded into four levels: no immunostaining (0), weak (1), moderate (2), and intense (3). The proportion of positive cells was scored as follows: 0 (none), 1 (1\%), 2 (2\%-10\%), 3 (11\%-33\%), 4 (34\%$66 \%)$, and $5(67 \%-100 \%)$. The sum index was obtained by totaling the scores of intensity and proportion of staining. If the final score was equal to or greater than 4 , the immunoreactivity was considered positive; otherwise, the immunoreactivity was considered negative. For negative controls, sections were treated in the same way, except that they were incubated with Tris-buffered saline instead of primary antibodies.

\section{Statistical analysis}

SPSS ver. 15.0 software (SPSS Inc., Chicago, IL, USA) was used for statistical analysis. Associations between the clinicopatho- logical factors and expression of antibodies were tested using a chi-square test. Univariate and multivariate Cox proportional regression analyses for overall survival and relapse-free survival were performed. A p-value of <.05 was considered statistically significant.

\section{RESULTS}

\section{Clinical features}

The 82 patients included 72 males and 10 females with a mean age of 57 years (range, 26 to 77 years). HCC formation was attributed to the presence of hepatitis $B$ virus (HBV) surface antigen in 64 patients, alcohol-related complication in seven patients, presence of anti-hepatitis $C$ virus antibody in four patients, and unknown etiology in seven patients. The surrounding liver tissue showed cirrhosis in $56 \%$ of the patients (46/82) and chronic hepatitis with varying degrees of fibrosis in the remaining $44 \%$ of the patients $(36 / 82)$.

\section{Expression of suggested CSC markers}

In cirrhotic livers, all four examined markers showed intense immunoreactivity in proliferating reactive bile ductules. These ductules are thought to originate from hepatic progenitor cells and thus were considered as an internal positive control. EpCAM expression in HCC tissues showed intense cytoplasmic and/or membranous staining (Fig. 1). In 82 HCC samples, 46 were EpCAM-positive (56\%) and five were K19-positive (6\%). Of the 82 HCCs, only two cases showed CD133 and CD 56 expression, respectively.

\section{Correlations between expression of suggested CSC markers and clinicopathological features}

Analysis of the relationships between expression of K19, CD133, and CD56 and clinicopathologic features was not meaningful, since the number of cases with K19, CD133, and CD56 expression was very small. However, EpCAM expression significantly correlated with higher histologic grade $(\mathrm{p}=.006)$, elevated AFP level ( $\mathrm{p}=.001)$, and microvessel invasion of the tumor cells $(\mathrm{p}=.030)$ (Table 2). Additionally, EpCAM expression was

Table 1. Summary of antibodies and conditions used for immunohistochemistry

\begin{tabular}{|c|c|c|c|c|c|}
\hline Antibody & Clone & Source & Dilution & Antigen retrieval & Antibody incubation \\
\hline EpCAM & VU-1D9 & Calbiochem & $1: 500$ & BOND ER solution I (pH 6.0), $100^{\circ} \mathrm{C}, 20 \mathrm{~min}$ & Room temperature, 20 min \\
\hline K19 & $\mathrm{BA} 17$ & DakoCytomation & $1: 100$ & BOND ER solution I (pH 6.0), $100^{\circ} \mathrm{C}, 20 \mathrm{~min}$ & Room temperature, 20 min \\
\hline CD56 & $123 C 3$ & Zymed Laboratories & $1: 400$ & BOND ER solution II (pH 9.0), $100^{\circ} \mathrm{C}, 20 \mathrm{~min}$ & Room temperature, $20 \mathrm{~min}$ \\
\hline
\end{tabular}

EpCAM, epithelial cell adhesion molecule; K19, keratin 19. 

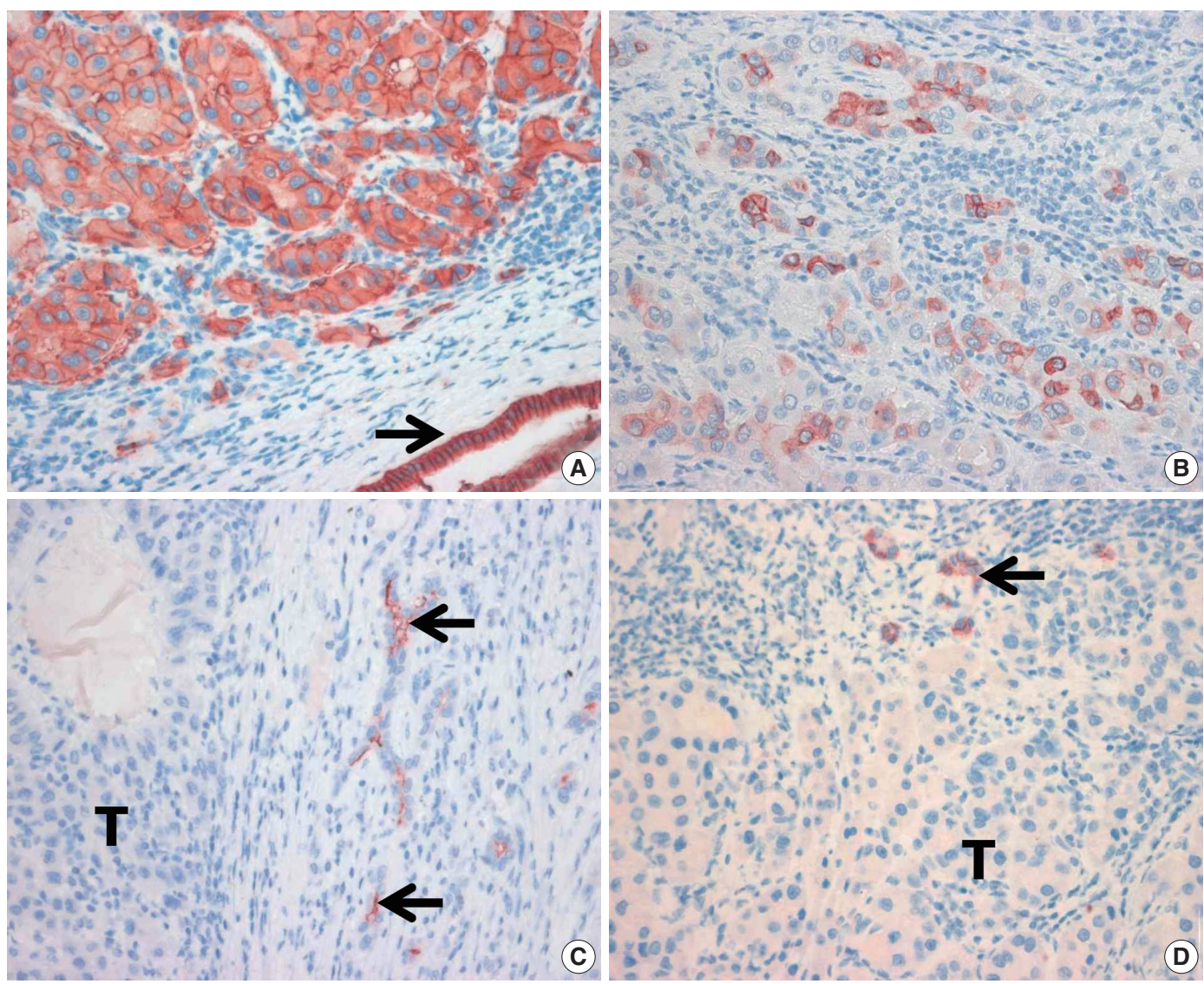

Fig. 1. (A) Membranous expression of epithelial cell adhesion molecule (EpCAM) in hepatocellular carcinoma cells. Bile duct epithelial cells also showed strong expression of EpCAM (arrow). (B) Tumor cells with cytoplasmic reactivity for keratin 19. Absence of CD133 (C) and CD56 (D) immunoreactivity in tumor cells (T). Note the positive immunoreactivity for CD133 and CD56 in bile ductular cells (arrows).

strongly associated with K19 expression $(\mathrm{p}=.041)$.

\section{Outcome}

In 82 patients with HCC, follow-up intervals ranged from 0.1 to 69 months (mean of 23 months). Eleven patients died, and 16 showed local recurrence or latent distant metastasis. The mean overall survival time of patients with EpCAM-expressing HCC was $57.2 \pm 3.7$ months, while that of EpCAM-negative HCC was $62.0 \pm 2.9$ months. The mean relapse-free survival time of patients with EpCAM-expressing HCC was $40.5 \pm 5.9$ months, while that of patients with EpCAM-negative HCC was $53.8 \pm$ 5.0 months. However, these results were not statistically significant. In univariate Cox regression analysis, $\mathrm{T}$ category signifcantly correlated with poor patient overall survival $(\mathrm{p}=.003)$. Additionally, T category, preoperative serum AFP level, and vascular invasion were associated with decreased relapse-free survival $(\mathrm{p}=.019, \mathrm{p}=.048$, and $\mathrm{p}=.011$, respectively). Multivariate analysis revealed that $\mathrm{T}$ category and vascular invasion were independent indicators of relapse $(\mathrm{p}=.011$ and $\mathrm{p}=.007$, respectively).

\section{DISCUSSION}

Carcinogenesis is currently explained using two models, one involving a traditional stochastic onset and a second based on the role of CSCs. According to the CSC model, only a small population of tumor cells has the ability to divide and repopulate within the tumor. ${ }^{73}$ CSCs are responsible for tumor initiation, maintenance, growth, metastasis, and relapse after therapy. ${ }^{3-6}$ The expression of several proposed CSC markers in HCC has been reported and might prove to be useful for predicting the prognosis of HCC patients. ${ }^{11,14-16}$ However, little is known about the relationships between the IHC expression of CSC markers in paraffin-embedded tissue sections and clinicopathologic factors or clinical outcomes of HCC.

EpCAM is a transmembrane intercellular protein that was initially posed as a homophilic cell adhesion molecule ${ }^{17}$ and is highly up-regulated in most human epithelial cancers, including HCC. ${ }^{14-16,18}$ EpCAM has been validated as a marker of stem cells in the liver, and EpCAM-positive HCC is likely to have 
Table 2. The relationship between the expression of suggested stem cell marker EpCAM and clinicopathologic characteristics

\begin{tabular}{|c|c|c|c|c|c|}
\hline \multirow{2}{*}{\multicolumn{2}{|c|}{ Characteristic }} & \multirow{3}{*}{$\begin{array}{l}\text { No. } \\
49\end{array}$} & \multicolumn{3}{|c|}{ EpCAM } \\
\hline & & & \multirow{2}{*}{$\begin{array}{c}\text { Positive } \\
30\end{array}$} & \multirow{2}{*}{$\begin{array}{c}\text { Negative } \\
19\end{array}$} & \multirow{2}{*}{$\frac{p \text {-value }}{.254}$} \\
\hline Age (yr) & $<60$ & & & & \\
\hline & $\geq 60$ & 33 & 16 & 17 & \\
\hline \multirow[t]{2}{*}{ Sex } & Female & 10 & 7 & 3 & .344 \\
\hline & Male & 72 & 39 & 33 & \\
\hline \multirow[t]{2}{*}{ AFP (ng/mL) } & $<100$ & 58 & 26 & 32 & .001 \\
\hline & $\geq 100$ & 24 & 20 & 4 & \\
\hline \multirow[t]{2}{*}{ Albumin (g/dL) } & $\geq 3.5$ & 79 & 45 & 34 & .418 \\
\hline & $<3.5$ & 3 & 1 & 2 & \\
\hline \multirow[t]{2}{*}{ PIVKA-II (mAU/mL) } & $<50$ & 26 & 25 & 1 & .798 \\
\hline & $\geq 50$ & 44 & 24 & 20 & \\
\hline \multirow[t]{3}{*}{ T category } & I & 34 & 16 & 18 & .230 \\
\hline & $\|$ & 36 & 24 & 12 & \\
\hline & III and IV & 12 & 6 & 6 & \\
\hline \multirow[t]{2}{*}{ Cirrhosis } & Absence & 36 & 24 & 12 & .088 \\
\hline & Presence & 46 & 22 & 24 & \\
\hline \multirow[t]{2}{*}{ Etiology } & Non-viral & 14 & 7 & 7 & .614 \\
\hline & Viral & 68 & 39 & 29 & \\
\hline \multirow[t]{2}{*}{ Microvessel invasion } & Absence & 39 & 17 & 22 & .030 \\
\hline & Presence & 43 & 29 & 14 & \\
\hline \multirow[t]{2}{*}{ Intrahepatic metastasis } & Absence & 67 & 36 & 31 & .362 \\
\hline & Presence & 15 & 10 & 5 & \\
\hline \multirow[t]{2}{*}{ Ascites } & Absence & 75 & 44 & 31 & .125 \\
\hline & Presence & 7 & 2 & 5 & \\
\hline \multirow[t]{2}{*}{ Histologic grade } & 1 and 2 & 50 & 22 & 28 & .006 \\
\hline & 3 and 4 & 32 & 24 & 8 & \\
\hline \multirow[t]{2}{*}{ K19 } & Negative & 77 & 41 & 36 & .041 \\
\hline & Positive & 5 & 5 & 0 & \\
\hline
\end{tabular}

EpCAM, epithelial cell adhesion molecule; AFP, $\alpha$-fetoprotein; PIVKA-II, protein induced by vitamin K absence/antagonist-II; K19, keratin 19.

originated from hepatic stem cells. ${ }^{8,15,16,19}$ In the present study, we observed that EpCAM expression significantly correlated with tumor progression factors of HCC, such as elevated AFP level, vascular invasion, and high tumor grade. These findings are in agreement with previous studies showing that expression level of EpCAM correlates with de-differentiation ${ }^{11}$ and vascular invasion and is associated with the high AFP level in HCC. ${ }^{20,21}$ The prognosis of patients with EpCAM-negative HCC is considered to be better than that of those with EpCAM-positive HCC. ${ }^{11,14-16,20,21}$ Although EpCAM expression was not found to be an independent predictor of survival in patients with HCC in this study, EpCAM expression was found to be associated with well-known unfavorable prognostic factors in HCC. Thus, the use of EpCAM expression as an unfavorable prognostic factor of HCC is reasonable. Furthermore, gene expression profile study has demonstrated that EpCAM-positive and AFP-positive HCCs have more aggressive behavior and poor clinical outcome, whereas EpCAM-negative and AFP-negative HCCs have good prognosis. ${ }^{20}$ Based on these observations, EpCAM has at- tracted considerable attention as a possible therapeutic target for patients with HCC. ${ }^{7,8,13}$ Taken together, our findings suggest that EpCAM is a critical player in facilitating vascular invasion of tumor cells, leading to de-differentiation in HCC with high serum AFP level, and might be useful for predicting the prognosis of HCC patients. In this study, a proportion of HCCs (56\%) expressed EpCAM, and EpCAM expression was associated with K19 expression. These findings are in agreement with previous reports showing that the expression of EpCAM and K19 was positively correlated. ${ }^{15,21}$ The proportion of tissues expressing EpCAM in this study was a little higher than in earlier series, which reported EpCAM in $15.9 \%-48.7 \%$ of HCCs. ${ }^{15-17,20-23}$ Kimura et al. ${ }^{23}$ have demonstrated that EpCAM expression was observed more often in HCC patients with HBV than in those with other etiologies, and the immunoreactivity of EpCAM has been found in up to $78 \%$ of HCC patients with HBV. The high proportion of HCC patients with HBV (74\%) examined in this study might be a possible explanation for the higher EpCAMpositive rate. It has been hypothesized that the transformation 
of hepatic stem/progenitor cells (maturation arrest theory) underlies the occurrence of HCC expressing CSC markers. However, the concept of the CSC model is still debated. The expression of CSC markers in HCC can develop as an acquisition of progenitor cell features during the de-differentiation of cancer cells (de-differentiation theory)., ${ }^{5,15} \mathrm{Kim}$ and Park ${ }^{24}$ have proposed that HCC expressing CSC markers should be designated as "HCC with stemness-related marker expression" in order to avoid the implication that these HCCs originated from hepatic stem/progenitor cells.

Either $\mathrm{CD} 133$ or $\mathrm{CD} 56$ expression in this study was rare. Only two of $82 \mathrm{HCC}$ specimens showed positive staining for CD133 and $\mathrm{CD} 56$, suggesting that $\mathrm{CD} 133$ and $\mathrm{CD} 56$ expression is rare in paraffin-embedded HCC tissues. It has been reported that $0 \%-88 \% 11,15,16,25$ of human HCC tissue samples express CD133, and the expression of which is negatively correlated with presence of HBV. ${ }^{26}$ Several IHC studies have demonstrated CD56 expression in $0 \%-9.7 \%$ of human HCCs. ${ }^{11,25,27,28}$ The reason for this high discrepancy of positive rate of $\mathrm{CD} 133$ and $\mathrm{CD} 56$ in HCC is unclear. In our study, we employed the bond polymer IHC staining method to avoid endogenous biotin contamination, and all four examined markers always showed strong immunoreactivity in reactive bile ductules, which were considered as internal positive controls. The discrepancy in the expression rates of $\mathrm{CD} 133$ and $\mathrm{CD} 56$ is not easily explained but might be related to the different criteria for positivity, the quality of tissue samples analyzed, and the unique antibodies and immunostaining methods used in different studies. Our negative results in analysis of $\mathrm{CD} 133$ and $\mathrm{CD} 56$ through the use of specific antibodies in an established automated IHC system does not exclude the presence of these markers and can be linked to the different etiologies according to geographic background. Additional investigations with a larger population of HCC and strict criteria for positive immunoreactivity are necessary to determine the expression and clinical implication of these markers.

In conclusion, we showed that the EpCAM expression in paraffin-embedded tissue sections of HCC is associated with tumor progression factors and might be useful for predicting the prognosis of HCC patients. The high proportion of EpCAM expression in HCCs and its associations with unfavorable prognostic factors of HCC provide a basis for further investigation of antiEpCAM-targeted therapy.

\section{Conflicts of Interest}

No potential conflict of interest relevant to this article was reported.

\section{Acknowledgments}

This work was supported by the National Research Foundation of Korea (NRF) grant funded by the Korean Government (MSIP) (No. 2008-0062279).

\section{REFERENCES}

1. Torre LA, Bray F, Siegel RL, Ferlay J, Lortet-Tieulent J, Jemal A. Global cancer statistics, 2012. CA Cancer J Clin 2015; 65: 87-108.

2. El-Serag HB, Marrero JA, Rudolph L, Reddy KR. Diagnosis and treatment of hepatocellular carcinoma. Gastroenterology 2008; 134: 1752-63.

3. Visvader JE. Cells of origin in cancer. Nature 2011; 469: 314-22.

4. Clarke MF, Dick JE, Dirks PB, et al. Cancer stem cells: perspectives on current status and future directions: AACR Workshop on cancer stem cells. Cancer Res 2006; 66: 9339-44.

5. Bomken S, Fiser K, Heidenreich O, Vormoor J. Understanding the cancer stem cell. Br J Cancer 2010; 103: 439-45.

6. Bjerkvig R, Tysnes BB, Aboody KS, Najbauer J, Terzis AJ. Opinion: the origin of the cancer stem cell: current controversies and new insights. Nat Rev Cancer 2005; 5: 899-904.

7. Marquardt JU, Factor VM, Thorgeirsson SS. Epigenetic regulation of cancer stem cells in liver cancer: current concepts and clinical implications. J Hepatol 2010; 53: 568-77.

8. Yamashita T, Ji J, Budhu A, et al. EpCAM-positive hepatocellular carcinoma cells are tumor-initiating cells with stem/progenitor cell features. Gastroenterology 2009; 136: 1012-24.

9. Durnez A, Verslype C, Nevens F, et al. The clinicopathological and prognostic relevance of cytokeratin 7 and 19 expression in hepatocellular carcinoma: a possible progenitor cell origin. Histopathology 2006; 49: 138-51.

10. Zhu Z, Hao X, Yan M, et al. Cancer stem/progenitor cells are highly enriched in $\mathrm{CD} 133+\mathrm{CD} 44+$ population in hepatocellular carcinoma. Int J Cancer 2010; 126: 2067-78.

11. Chan AW, Tong JH, Chan SL, Lai PB, To KF. Expression of stemness markers (CD133 and EpCAM) in prognostication of hepatocellular carcinoma. Histopathology 2014; 64: 935-50.

12. Edge SB, Byrd DR, Compton CC, Fritz AG, Greene FL, Trotti A. AJCC cancer staging manual. 7th ed. New York: Springer, 2010.

13. Visvader JE, Lindeman GJ. Cancer stem cells: current status and evolving complexities. Cell Stem Cell 2012; 10: 717-28.

14. Yang $X R, X u$ Y, Yu B, et al. High expression levels of putative hepatic stem/progenitor cell biomarkers related to tumour angiogenesis and poor prognosis of hepatocellular carcinoma. Gut 2010; 59: 953-62.

15. Kim H, Choi GH, Na DC, et al. Human hepatocellular carcinomas with "Stemness"-related marker expression: keratin 19 expression 
and a poor prognosis. Hepatology 2011; 54: 1707-17.

16. Guo Z, Li LQ, Jiang JH, Ou C, Zeng LX, Xiang BD. Cancer stem cell markers correlate with early recurrence and survival in hepatocellular carcinoma. World J Gastroenterol 2014; 20: 2098-106.

17. Litvinov SV, Balzar M, Winter MJ, et al. Epithelial cell adhesion molecule (Ep-CAM) modulates cell-cell interactions mediated by classic cadherins. J Cell Biol 1997; 139: 1337-48.

18. Went PT, Lugli A, Meier S, et al. Frequent EpCAM protein expression in human carcinomas. Hum Pathol 2004; 35: 122-8.

19. Breuhahn K, Baeuerle PA, Peters M, et al. Expression of epithelial cellular adhesion molecule (Ep-CAM) in chronic (necro-)inflammatory liver diseases and hepatocellular carcinoma. Hepatol Res 2006; 34: 50-6.

20. Yamashita T, Forgues M, Wang W, et al. EpCAM and alpha-fetoprotein expression defines novel prognostic subtypes of hepatocellular carcinoma. Cancer Res 2008; 68: 1451-61.

21. Govaere O, Komuta M, Berkers J, et al. Keratin 19: a key role player in the invasion of human hepatocellular carcinomas. Gut 2014; 63: 674-85.

22. Shan YF, Huang YL, Xie YK, et al. Angiogenesis and clinicopathologic characteristics in different hepatocellular carcinoma subtypes defined by EpCAM and alpha-fetoprotein expression status. Med Oncol 2011; 28: 1012-6.

23. Kimura O, Kondo Y, Kogure T, et al. Expression of EpCAM increases in the hepatitis B related and the treatment-resistant hepatocellular carcinoma. Biomed Res Int 2014; 2014: 172913.

24. Kim H, Park YN. Hepatocellular carcinomas expressing 'stemness'related markers: clinicopathological characteristics. Dig Dis 2014; 32: 778-85.

25. Zen C, Zen Y, Mitry RR, et al. Mixed phenotype hepatocellular carcinoma after transarterial chemoembolization and liver transplantation. Liver Transpl 2011; 17: 943-54.

26. Yeh CT, Kuo CJ, Lai MW, et al. CD133-positive hepatocellular carcinoma in an area endemic for hepatitis B virus infection. BMC Cancer 2009; 9: 324.

27. Tsuchiya A, Kamimura H, Takamura M, et al. Clinicopathological analysis of CD133 and NCAM human hepatic stem/progenitor cells in damaged livers and hepatocellular carcinomas. Hepatol Res 2009; 39: 1080-90.

28. Tsuchiya A, Kamimura H, Tamura Y, et al. Hepatocellular carcinoma with progenitor cell features distinguishable by the hepatic stem/progenitor cell marker NCAM. Cancer Lett 2011; 309: 95-103. 